\title{
Prediction of efficacy of neoadjuvant chemoradiotherapy for rectal cancer: the value of texture analysis of magnetic resonance images
}

\author{
Zhenyu Shu $^{1} \cdot$ Songhua Fang ${ }^{1} \cdot$ Qin $\mathrm{Ye}^{2} \cdot$ Dewang Mao ${ }^{1} \cdot$ Hongfeng $\mathrm{Cao}^{3} \cdot$ Peipei Pang ${ }^{4,5} \cdot$ Xiangyang Gong $^{1,6}$
}

Published online: 9 March 2019

(c) The Author(s) 2019

\begin{abstract}
Purpose To explore the clinical feasibility of predicting the efficacy of neoadjuvant chemoradiotherapy (nCRT) for rectal cancer on the basis of texture analysis (TA) of T2-weighted imaging (T2WI).

Methods The cohort for this prospective study comprised 136 patients with rectal cancer to be treated with nCRT, all of whom underwent three MR scans (pre-, early, and post-nCRT). Treatment efficacy was assessed on the basis of the outcomes of pathologic complete response (pCR) and non-pCR as determined by postoperative pathological examination. Extraction and analysis of texture features in T2WI of defined tumor regions were performed by AK software. Pre- and early-nCRT texture features were selected as potential predictors of outcomes by logistic regression analysis, and a prediction model for $\mathrm{pCR}$ was developed. A receiver operating characteristic (ROC) curve was used to assess the predictive power of texture features in pre- and early-nCRT images.

Results Univariate logistic regression analysis demonstrated that the pre-nCRT features of energy, entropy, and skewness, and early-nCRT features of variance, kurtosis, energy, and entropy were independent predictors of pCR. A prediction model incorporating these predictors was constructed by multivariate logistic regression, The AUCs of pre-nCRT, early, and combined models were $0.751,0.831$, and 0.873 , respectively; the sensitivities 66,71 , and $75 \%$, respectively; and the specificities $87.22,86.11$, and $91.67 \%$, respectively.
\end{abstract}

Conclusions TA of T2WI images can predict the efficacy of nCRT for rectal cancer, possibly providing a new marker of tumor biological response in clinical practice.

Keywords Rectal tumor $\cdot$ Magnetic resonance imaging $\cdot$ Texture analysis $\cdot$ Neoadjuvant chemoradiotherapy

Xiangyang Gong

cjr.gxy@hotmail.com

$1 \quad$ Department of Radiology, Zhejiang Provincial People's Hospital, Affiliated People's Hospital of Hangzhou Medical College, NO. 158 Shangtang Road, Hangzhou, Zhejiang, China

2 Second Clinical College, Zhejiang Chinese Medical University, Hangzhou, China

3 Department of Anorectal, Zhejiang Provincial People's Hospital, Affiliated People's Hospital of Hangzhou Medical College, Hangzhou, China

4 Department of Radiotherapy, Zhejiang Provincial People's Hospital, Affiliated People's Hospital of Hangzhou Medical College, Hangzhou, China

5 GE Healthcare China, Shanghai, China

6 Institute of Artificial Intelligence and Remote Imaging, Hangzhou Medical College, Hangzhou, China

\section{Introduction}

Rectal cancer, one of the commonest malignant tumors of the digestive tract, has caused increasing morbidity in the past few years [1]. Its treatment outcomes have been significantly improved by neoadjuvant chemoradiotherapy (nCRT) [2], which can result in pathological complete remission (pCR) [3]. It also greatly reduces rates of local recurrence and distant metastasis of rectal cancer [4]. American cancer network guidelines state that concurrent preoperative nCRT and not surgical treatment alone should be the first choice for advanced rectal cancer [5]. However, there are significant individual variations in response to preoperative nCRT, and ineffective treatment may result in unjustified toxicity and a delay in more effective treatment $[6,7]$. Therefore, early identification (before treatment or 2-3 weeks after starting nCRT) of patients who may benefit from nCRT is vital [8]. 
Magnetic resonance imaging (MRI) has been designated as the primary imaging method for preoperative evaluation of rectal cancer by National Comprehensive Cancer Network (NCCN), because of its inherent advantages of high resolution in soft tissue and multiparametric imaging techniques [9]. MR T2-weighted imaging (T2WI) is widely used to assess the degree of tumor regression on the basis of morphological changes. Taylor et al. have reported that more than $70 \%$ of tumor volume changes detected by T2WI denote tumor responses [10] as determined by morphological evaluation by MRI. However, these findings may underestimate the difficulty of determining the margin between residual tumor and surrounding normal tissues and of differentiating between residual tumor and fibrosis after nCRT, on the basis of morphological changes only. More sensitive and reliable MRI biomarkers are needed to evaluate the efficacy or predict early responses to treatment in clinical practice.

Recently, a new imaging biomarker, texture analysis (TA) has been used to determine image eigenvalues of spatial-temporal heterogeneity in tumor imaging. TA has been used to assess the severity, progression, and response to therapy of quite a few diseases [11-14]. A preliminary study demonstrated that TA based on MRI can be used to evaluate the response of patients with rectal cancer to nCRT [7]. However, to the best of our knowledge, texture features at different intervals after nCRT have not been studied.

Accordingly, the purpose of this study was to identify texture features on T2WI images at different time points that are useful for evaluating response to nCRT in patients with rectal cancer.

\section{Materials and methods}

\section{Patients and study design}

This study was approved by the Ethics Committee of Zhejiang Provincial People's Hospital, and written informed consent was obtained from all patients. From February 2014 to April 2017, 177 consecutive patients were prospectively enrolled. All participants had histologically proven rectal adenocarcinoma and locally advanced tumor stage II (cT34, N0, M0) or III (cT1-4, N+, M0). Inclusion criteria were as follows: lower margin of tumor less than $12 \mathrm{~cm}$ from the anal verge on rectal examination; normal preoperative hepatorenal function, routine blood tests, and cardiopulmonary function; no contraindications to chemotherapy or surgery; no preoperative severe heart, lung, or cerebrovascular disease; and no evidence of distant metastases. Forty-one of the original cohort population were excluded for the following reasons: seven patients did not undergo three magnetic resonance examinations, five did not complete nCRT, 17 patients did not complete surgery, two lacked postoperative pathological results, and 10 had MRI motion artifacts. Therefore, a total of 136 patients were included in this study. A flowchart of patient enrollment is shown in Fig. 1.

\section{MRI studies}

All enrolled patients were scanned by a 3.0T MR (Discovery MR 750; GE Healthcare, Waukesha, WI, USA). Axial T2WI FR-FSE sequences were used. The slice thickness was $3 \mathrm{~mm}$; interval: $0.3 \mathrm{~mm}$; TR/TE: $350-5000 \mathrm{~ms} / 115 \mathrm{~ms}$; FOV: $18 \mathrm{~cm} \times 18 \mathrm{~cm}$; pixel matrix: $256 \times 256$; and ETL: 20 . Images were acquired in three directions, sagittal, oblique coronal (parallel to the long axis of the rectum), and oblique axial (perpendicular to the long axis of the tumor). After that, axial SE DWI echo-planar imaging sequences with background body signal suppression were acquired at $b$ values of 0 and $800 \mathrm{~s} / \mathrm{mm}^{2}$. Subsequently, axial three-dimensional LAVA DCE-MRI images were acquired. The first MRI examination was performed to assess tumor stage one day prior to commencing nCRT, the second MRI to assess early-treatment response in the third week of nCRT, and the third to monitor the response at the end of nCRT. Between 6 and 8 weeks after undergoing nCRT, patients underwent total mesorectal excision (TME) performed by an experienced abdominal surgeon (more than 10 years of experience). As our study aimed at prediction and early assessment of tumor response to nCRT, the focus was on analyzing preand early-MR findings.

\section{Neoadjuvant chemoradiotherapy}

The nCRT comprised radiotherapy at a dose of $2.0 \mathrm{~Gy} /$ day, 5 days per week for 5 weeks, the total dose being $50 \mathrm{~Gy}$ delivered in one posterior and two lateral positions. Chemotherapy was administered simultaneously with the radiotherapy and comprised intravenous oxaliplatin at $50 \mathrm{mg} / \mathrm{m}^{2}$ per week and oral capecitabine at $825 \mathrm{mg} / \mathrm{m}^{2}$ per day, 5 days per week for 5 weeks. All enrolled patients successfully completed the planned nCRT.

\section{Postoperative pathological evaluation}

Surgical excision specimens were evaluated by a pathologist (5 years' experience). Pathological grading was conducted according to the following criteria for histopathology after radiochemotherapy proposed by Dworak et al. [15]: tumor Regression Grade 4, TRG4-tumor cells have been completely eradicated, with no viable tumor cells observed and fibrosis only being observed; TRG3-tumor cells appear to be absent, more than $50 \%$ of tumor cells appear to be degenerate, and there is accompanying obvious fibrosis of tumor tissue; TRG2-there is a moderate reduction in the number of tumor cells and significant fibrosis in $26-50 \%$ of 
Fig. 1 Flowchart of the study

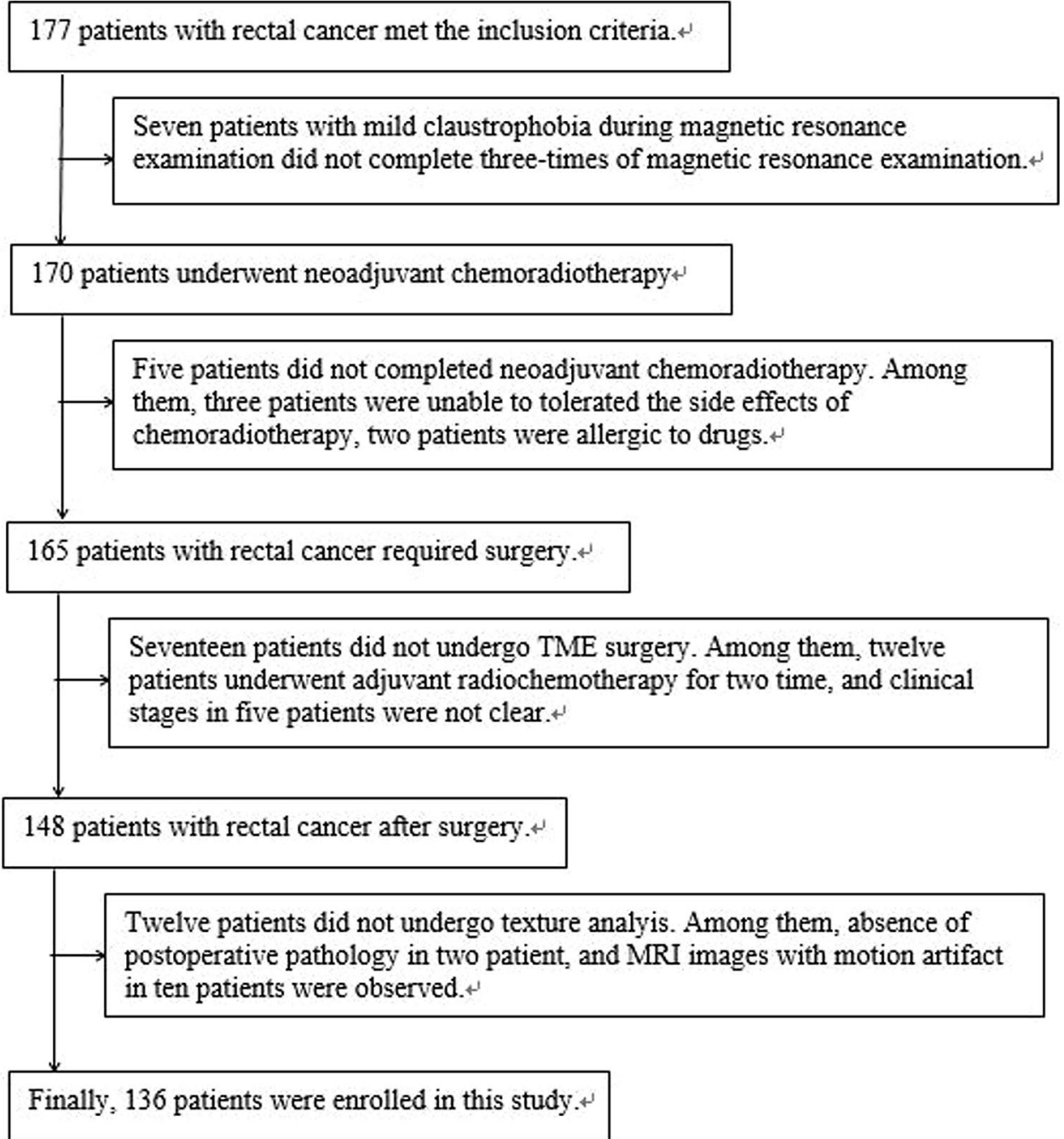

tumor tissue; TRG1 - the absence of morphological change in tumor tissue and significant fibrosis in less than $25 \%$ of tumor tissue; and TRG0-no reduction in the number of tumor cells. Modified TRG categories based on Dworak et al.'s definition were used to separate the patients into pathologic complete response (pCR; TGR4) and non-pCR groups (all other TRG categories).

\section{Image segmentation and extraction of features}

Manual segmentation of the MRI images was performed independently by two radiologists (A and B, with 7 and 10 years of experience in abdominal MRI interpretation, respectively). Regions of interest (ROI) were placed at the tumors and manually delineated to cover the largest tumor regions of the rectal cancers. DWI and LAVA were used as references to ensure accurate ROI positioning (Fig. 2). The radiologists were blinded to the histopathological findings. If there was no definite residual tumor after nCRT, especially in the case of complete remission, the ROI was placed on the intestinal wall furthest from the initial tumor.

Within the ROIs, histogram features were extracted using the AK software (Anolysis Kit, GE Healthcare, China). Features were extracted independently by the same two radiologists who performed image segmentation in a blinded fashion. Interobserver reproducibility of extraction of features between these two radiologists was initially analyzed. In addition, to assess intraobserver reproducibility, Radiologist A repeated the features extraction twice during a 2-week period following the same procedure. The following histogram features were extracted:

Variance

variance $=\frac{1}{N-1} \sum_{i=1}^{N}(X(i)-\bar{X})^{2}$,

where $\bar{X}$ is the mean of $X$. 
Fig. 2 a, $\mathbf{b}$ The ROI ranges of the tumors pre- and early-nCRT in T2WI images, respectively. c, d The tumor regions extracted by AK software
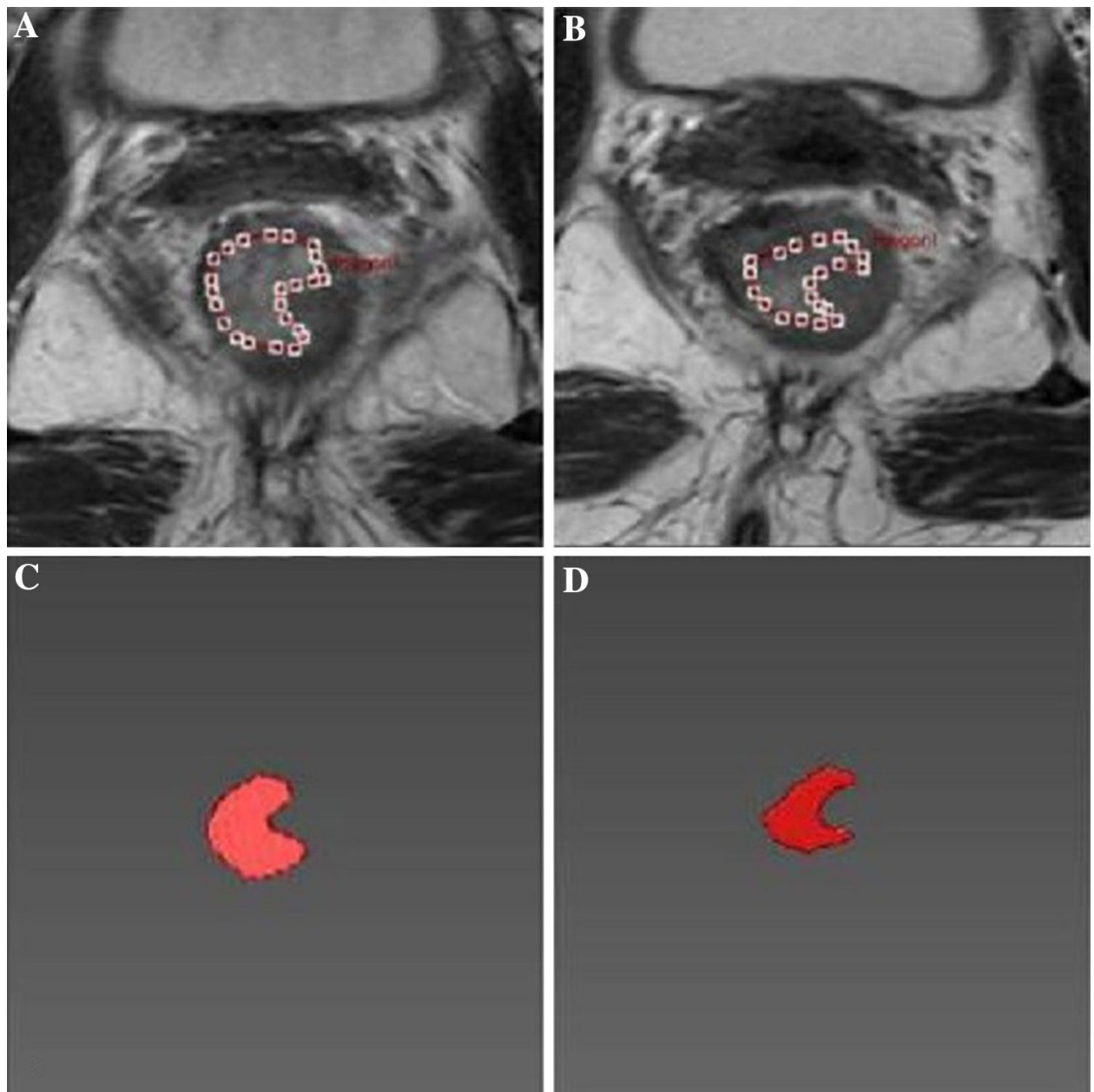

Skewness

skewness $=\frac{\frac{1}{N} \sum_{i=1}^{N}(X(i)-\bar{X})^{3}}{\left(\sqrt{\frac{1}{N} \sum_{i=1}^{N}(X(i)-\bar{X})^{2}}\right)}$,

where $\bar{X}$ is the mean of $X$.

Kurtosis

kurtosis $=\frac{\frac{1}{N} \sum_{i=1}^{N}(X(i)-\bar{X})^{4}}{\left(\sqrt{\frac{1}{N} \sum_{i=1}^{N}(X(i)-\bar{X})^{2}}\right)}$,

where $\bar{X}$ the mean of $X$.

Uniformity

uniformity $=\sum_{i=1}^{N_{l}} P(i)^{2}$
Energy

energy $=\sum_{i}^{N} X(i)^{2}$

Entropy

entropy $=-\sum_{i=1}^{N_{l}} P(i) \log _{2} P(i)$

\section{Statistical analysis}

All data were analyzed using SPSS 20.0 and MedCalc software. Enumeration data are expressed as a percentage, and measurement data as mean \pm standard deviation (SD). Kolmogorov-Smirnov was used to test data normality. Texture features were compared between pCR and non-pCR at different time points by independent sample $t$ test. The texture features were also compared between the two groups at the same time points by independent sample $t$-test. $P<0.05$ was considered to denote statistical significance. The texture 
features that differed significantly between different time points and at the same time point were selected. Cutoff values for these features were determined and classified into two categories by receiver-operating characteristic (ROC) curves, after which the texture features at the times of preand early nCRTs were analyzed to predict $\mathrm{pCR}$ group by multifactor logistic regression analysis. The same method was used to predict the pCR group by the combined features of pre- and early nCRTs. The constructed model predicts the pCR group, and the ROC curves were used to evaluate the diagnostic performance.

Table 1 Clinical pathology information of patients in this study

\begin{tabular}{llc}
\hline Characteristic & Classification & No. $(\%)$ \\
\hline Gender & Male & $84(62)$ \\
Median age(year) & Female & $52(38)$ \\
Tumor location & $\leq 60$ & $80(59)$ \\
& $>60$ & $56(41)$ \\
clinical T stage & Low-rectum & $76(56)$ \\
& Mid-rectum & $60(44)$ \\
clinical N stage & T2 & $24(18)$ \\
& T3 & $84(61)$ \\
Pathologic TGR scores & T4 & $28(21)$ \\
& N0 & $24(18)$ \\
& N+ & $112(82)$ \\
& TRG-0 & $12(9)$ \\
& TRG-1 & $36(26)$ \\
& TRG-2 & $28(21)$ \\
& TRG-3 & $24(18)$ \\
& TRG-4 & $36(26)$ \\
\hline
\end{tabular}

\section{Results}

\section{Clinical characteristics and results of neoadjuvant radiochemotherapy}

The 136 study patients comprised 84 men and 52 women of mean age 59.3 years (33-77 years). Seventy-six of these patients had low rectal (56\%) and 60 middle rectal adenocarcinoma (44\%). TRG4 was achieved in 36 patients (26\%), TRG3 in $24(18 \%)$, TRG2 in $28(21 \%)$, TRG1 in $36(26 \%)$, and TRG0 in 12 (9\%). Thus, the 36 patients who achieved TRG4 grade had pathological complete responses (pCR group), the remainder comprising the non-pCR group $(n=100)$. Detailed clinical characteristics are listed in Table 1.

\section{Interobserver and intraobserver reproducibility of extraction of radiomics features}

The intraobserver ICC calculated from Radiologist A's two sets of measurements $0.753-0.914$. The interobserver agreement between Radiologist A's first measurements and Radiologist B's measurements were in the range of 0.724-0.898, indicating acceptable intra- and interobserver reproducibility of extraction of features.

\section{Statistical analysis of texture values at different and the same time points of neoadjuvant chemoradiotherapy}

The texture features of skewness, entropy, and energy of prenCRT and of variance, kurtosis, energy, and entropy of early nCRT were differed significantly between the PCR and nonPCR groups; in addition, in the $\mathrm{PCR}$ group these texture features differed significantly between the pre-nCRT and early-nCRT periods. Details are presented in Table 2 and Figs. 3 and 4.

Table 2 Statistical analysis of texture values in two groups at each time point

\begin{tabular}{lllllc}
\hline Time point & PCR $(n=36)$ & Non-pCR $(n=100)$ & $P$ & PCR $(n=36)$ & Non-pCR $(n=100)$ \\
\hline & Variance & & & Kurtosis & \\
Pre-nCRT & $3250 \pm 1236$ & $3036 \pm 1128$ & 0.633 & $3.165 \pm 0.846$ & $3.871 \pm 1.694$ \\
Early nCRT & $1899 \pm 552^{*}$ & $2522 \pm 827$ & 0.045 & $4.800 \pm 1.288^{*}$ & $3.206 \pm 1.211$ \\
$P$ & 0.012 & 0.847 & & 0.006 & 0.127 \\
& Energy & & & Entropy & 0.243 \\
Pre-nCRT & $0.0084 \pm 0.0025^{*}$ & $0.0115 \pm 0.0033$ & 0.016 & $7.109 \pm 0.367^{*}$ & $6.823 \pm 0.309$ \\
Early nCRT & $0.0147 \pm 0.0051^{*}$ & $0.0106 \pm 0.0046$ & 0.035 & $5.436 \pm 0.934^{*}$ & $6.803 \pm 0.577$ \\
$P$ & 0.005 & 0.356 & & 0.005 & 0.282 \\
& Skewness & & & Uniformity & 0.03 \\
Pre-nCRT & $0.2281 \pm 0.5326^{*}$ & $0.7002 \pm 0.5914$ & 0.043 & $1.3107 \pm 0.9628$ & $1.6095 \pm 1.1727$ \\
Early nCRT & $0.5611 \pm 0.1469$ & $0.4582 \pm 0.4395$ & 0.053 & $1.5511 \pm 1.0637$ & $1.3643 \pm 1.3653$ \\
$P$ & 0.043 & 0.372 & & 0.592 & 0.012 \\
\hline
\end{tabular}

*Showed statistical differences between pCR and non-pCR, and there is also a statistical difference between pre-nCRT and early nCRT 

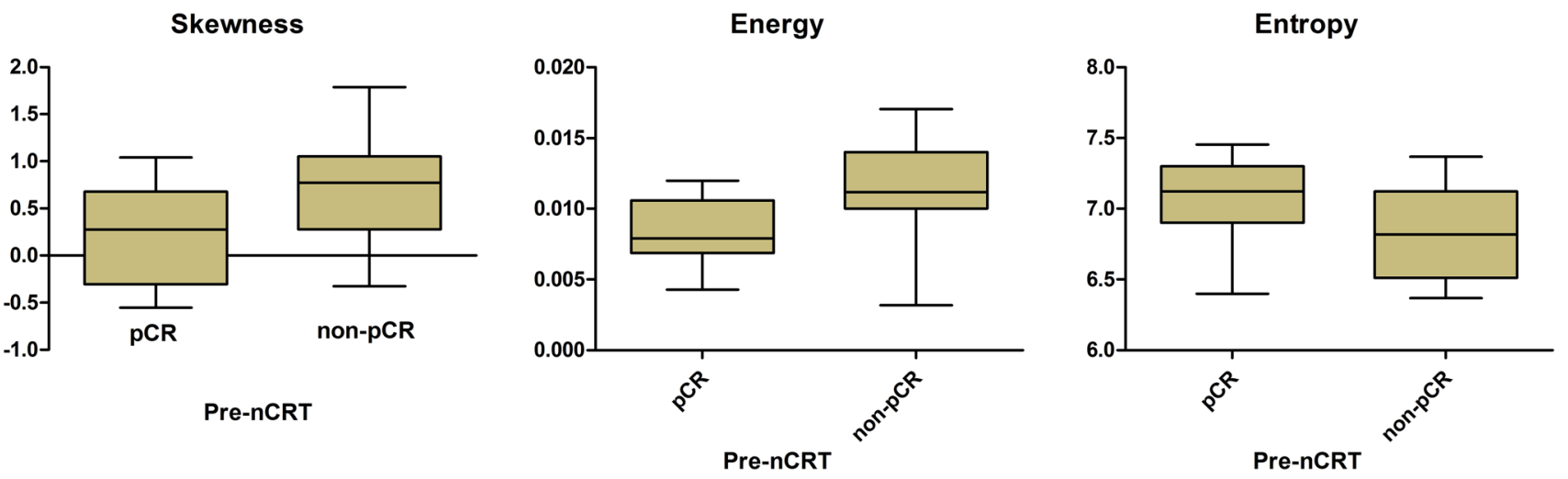

Fig. 3 Box plot showing the indicated features of pre-nCRT differing significantly between the pCR and non-pCR groups

Fig. 4 Box plot showing the indicated features of early nCRT differing significantly between the pCR and non-pCR groups

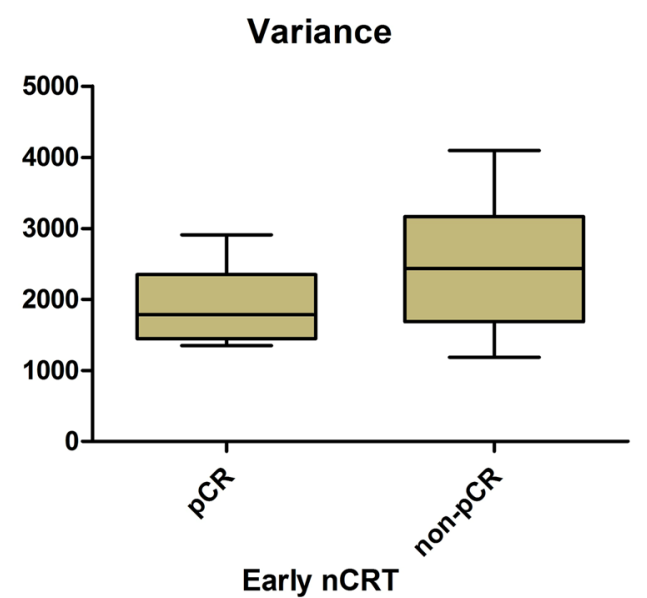

Energy

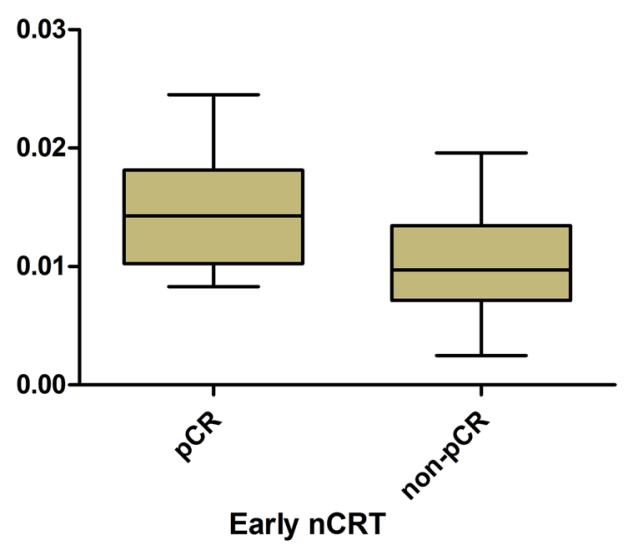

\section{Kurtosis}

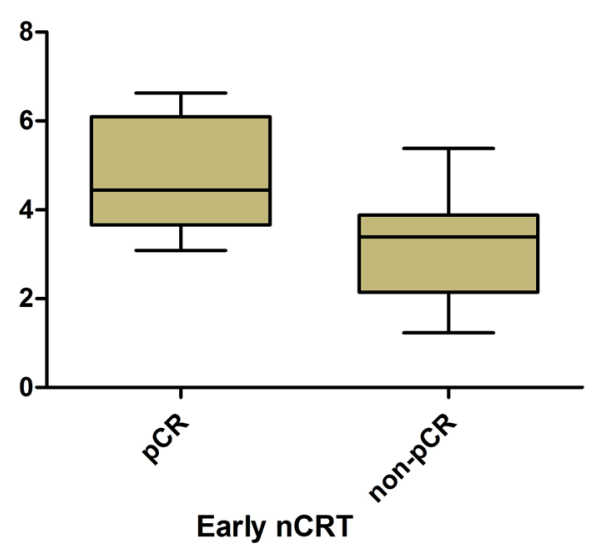

Entropy

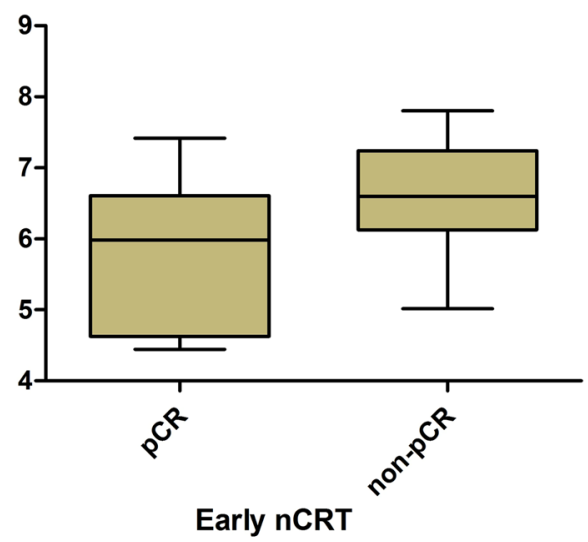

Diagnostic efficiency of texture features that differed significantly between pre- and early nCRTs

The cutoff values for entropy, energy, and skewness in the pre-nCRT period were $7.07763,0.00919$, and 0.30657 , respectively, and the AUC 0.729, 0.751, and 0.733, respectively. The cutoff values for kurtosis, variance, entropy, and energy in the early-nCRT period were $3.99289,2005.16$, 5.98335, and 0.012682, respectively, and AUC 0.809, 0.72, 0.744 , and 0.716 , respectively. Full results are listed in Table 3 and Fig. 5a, b. 
Table 3 Diagnostic significance of texture values analyzed by ROC curve

\begin{tabular}{lllll}
\hline Texture value & Critical value & AUC & $\begin{array}{l}\text { Sensitiv- } \\
\text { ity (\%) }\end{array}$ & Specificity (\%) \\
\hline Pre-entropy & $\leq 7.07763$ & 0.729 & 66 & 63.63 \\
Pre-energy & $>0.00919$ & 0.751 & 80 & 72.81 \\
Pre-skewness & $>0.30657$ & 0.733 & 76 & 68.56 \\
Early kurtosis & $\leq 3.99289$ & 0.809 & 84 & 77.25 \\
Early variance & $>2005.16$ & 0.72 & 72 & 72.19 \\
Early entropy & $>5.98335$ & 0.744 & 92 & 58.43 \\
Early energy & $\leq 0.012682$ & 0.716 & 72 & 66.67 \\
\hline
\end{tabular}

\section{Logistic regression analyses of prediction of PCR}

In the pre-nCRT period, energy was identified as an independent predictor of pCR by multifactor logistic regression analysis. For distinguishing between the pCR and non-pCR groups by energy, the AUC was 0.751 , sensitivity $66 \%$, and specificity $87.22 \%$. Multivariate analysis revealed that independent predictors of $\mathrm{pCR}$ comprised kurtosis, entropy, and energy in the early-nCRT period; ROC curves to determine the efficacy of these features in predicted pCR showing an AUC of 0.831, sensitivity of $71 \%$, and specificity of $86.11 \%$. A new model was then built that combined all the independent risk factors in the pre- and early-nCRT periods, for this the AUC was 0.873 ,

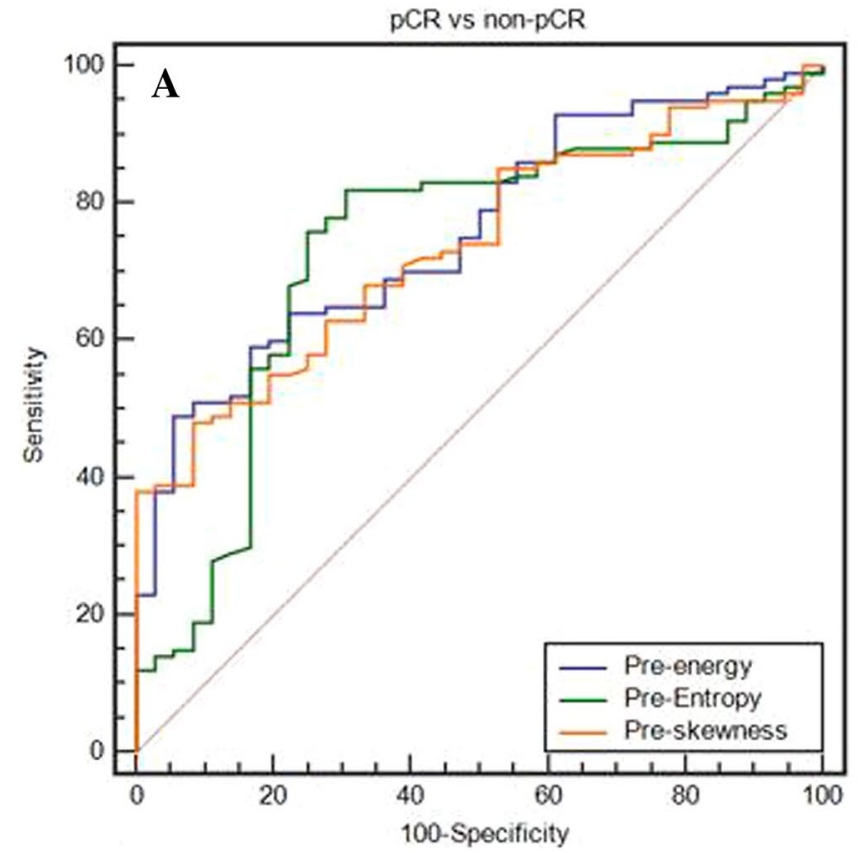

Fig. 5 a ROC curves analyzing the discriminatory power of skewness, energy, and entropy in pre-CRT in distinguishing between pCR and non-pCR. b ROC curves analyzing the discriminatory power of sensitivity $75 \%$, and specificity $91.67 \%$. Full results are listed in Table 4 and Fig. 6.

\section{Discussion}

Our results show that texture analysis based on T2WI images can predict sensitivity to nCRT of patients with rectal cancer. In particular, a combination of pre- and early-treatment texture features were identified as the best independent predictors for distinguishing between the $\mathrm{pCR}$ and non-pCR groups, potentially enabling adjustment of treatment and timely planning.

TA refers to a variety of image processing methods that seek to describe the relationships between the gray-level intensity of pixels and their positions within an image [16]. In tumor imaging, TA allows for quantitation of intratumoral heterogeneity based on the distribution of gray-level values (histogram variables) within a given ROI. Heterogeneity of malignant lesions is known to correlate with tumor aggressiveness. TA has recently been recognized as a promising tool in cancer research. In particular, it has been shown to be of value in predicting the response of rectal cancer to nCRT $[17,18]$. However, the stability of such predictions remains unsatisfactory. More reliable means of predicting response of rectal cancer to nCRT would confer a significant leverage in determining the optimal line of treatment for these patients.

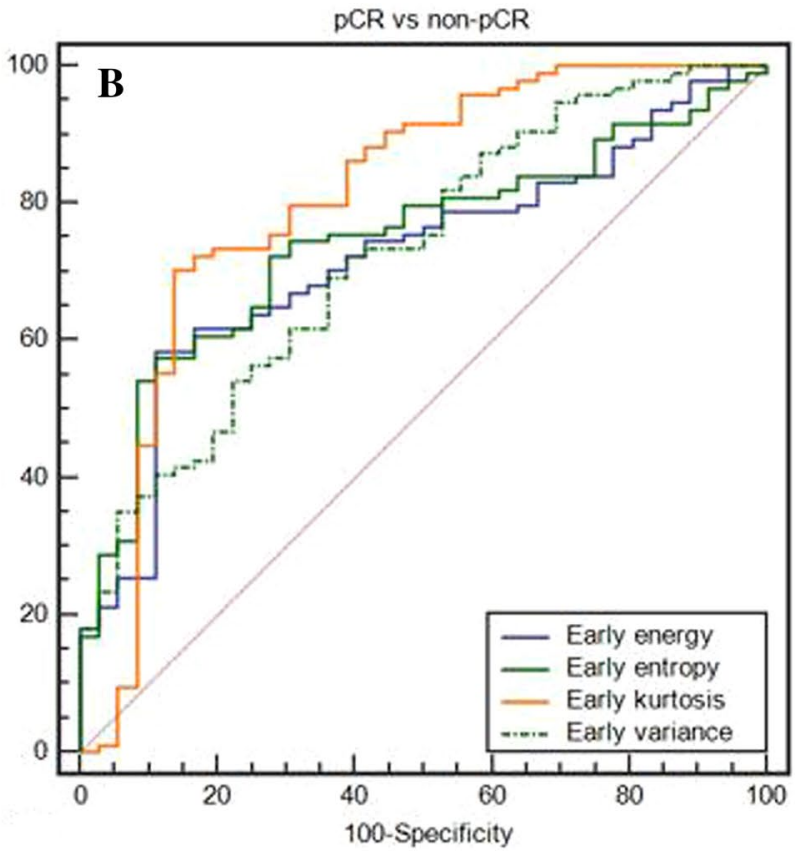

variance, kurtosis, energy, and entropy in early CRT in distinguishing between $\mathrm{pCR}$ and non-pCR 


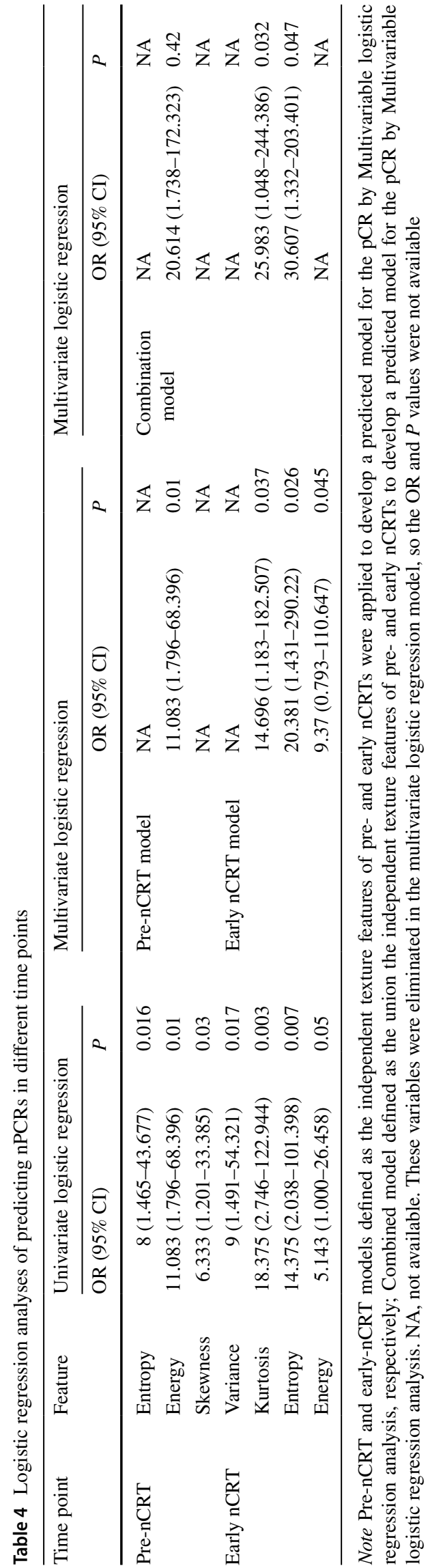

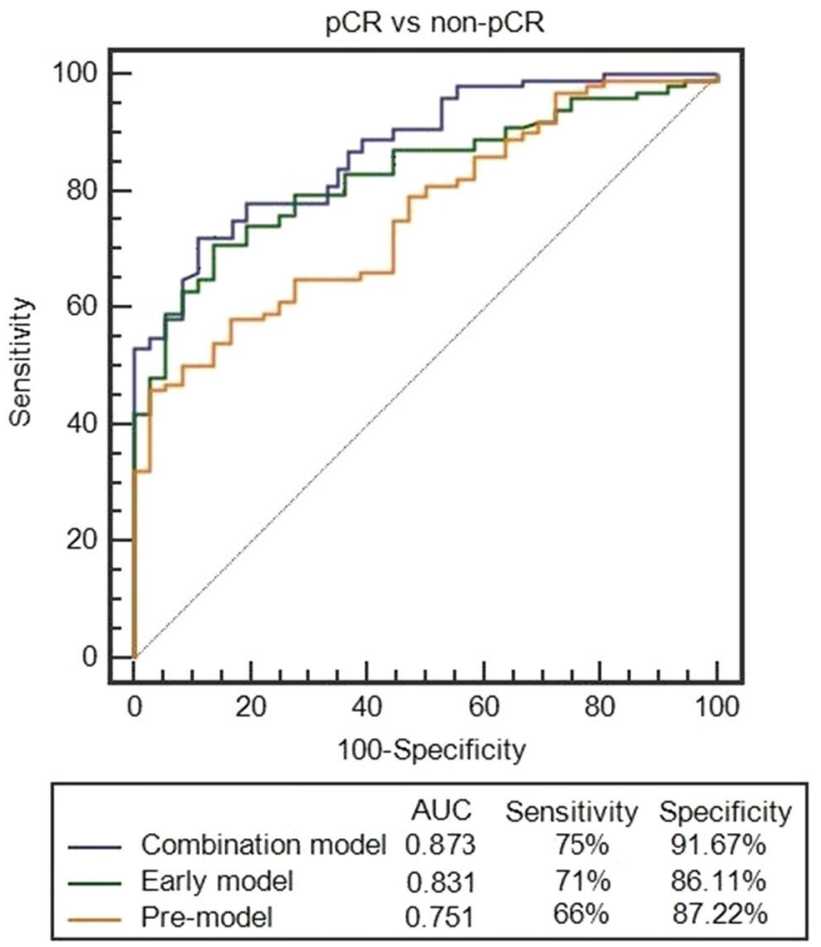

Fig. 6 ROC curves analyzing the discriminatory powers of the three models in distinguishing between pCR and non-pCR

Our results demonstrate the ability of TA assessed at different time points to provided quantitative measures for predicting response of rectal cancer to nCRT; we found that energy, entropy, and skewness in the pre-nCRT period are independent predictors of tumor response. The identification of entropy and energy is consistent with the findings reported by Meng et al. [19]; however, they did not identify skewness. We speculate that this inconsistency may be associated with the use of different analysis software and also the baseline characteristics of the participants. Theoretically, skewness reflects asymmetry of the distribution of gray-level intensity of pixels within a given ROI: higher values of skewness reflecting greater complexity of the ROI texture and indicating greater heterogeneity of a lesion [20]. Our study included a higher proportion of patients with considerable tumor heterogeneity, which indicates greater skewness. Thus, the skewness may be more suitable for predicting response to nCRT of tumors, which found pretreatment to be highly heterogeneous.

We also identified kurtosis, variance, entropy, and energy as independent predictors in early treatment, kurtosis being the best of these, having an AUC of 0.809. This finding is in agreement with De Cecoo et al.'s report that kurtosis is the best predictor in the pre-nCRT period [21]. Kurtosis reflects peakedness and tailedness of the histogram and is related inversely to the number of features included [22]; rectal cancers with smaller peaks have lower rates of response to 
treatment [23]. Thus, changes in kurtosis at an early stage of treatment may best reflect changes in tumor heterogeneity, potentially meaning that kurtosis should be the main feature for predicting response of rectal cancer to early nCRT. In addition, in our study, we used T2WI images for feature extraction on the basis of the results of De Cecoo et al.'s study result. T2WI offers a high signal-to-noise ratio, spatial resolution, and soft tissue contrast [24]. Also, MRI under high field intensity reduces the influence of noises, especially those biological heterogeneities that affect texture analysis [25]. Accordingly, texture analysis of high resolution images could reveal subtle alterations in the structure of the tissue caused by cancer. On the other hand, T2WI images enable tumor grading, aiding radiologists, and oncologists in selecting treatment. Thus, histogram analysis based on T2WI images may be a more useful aid to making treatment decisions.

In addition, we used a logistic regression model to perform texture analysis at different time points to maximize the stability of our prediction models. Prediction models in pre- and early nCRTs separately, as well as in a combination of these time periods, we found that both energy and entropy are independent predictors. In the pCR group, entropy was significantly higher before treatment than in early nCRT, indicating that treatment had an effect on tumor regression. These results are consistent with other's findings regarding a decrease in entropy values with reduction in a tumor's malignancy [26]. After nCRT, rectal tumor tissue demonstrates varying degrees of coexistence of fiber tissues with viable tumor cells; entropy reflects the intensity of spatial distribution [27] and has been found to be more sensitive to image changes than other texture features. These findings also indicate that after treatment tumor tissue in the pCR group displays the simplest internal change, that is, complete fibrosis. Energy may reflect the homogeneity of the normal tissue in an image. Greater changes in cells and tissues in response to treatment may correlate with the lower heterogeneity in pCR [28]. Subsequently, the inhomogeneity in an image would be further decreased, making changes in energy the most sensitive indicator of this process and explaining why energy and entropy are both independent predictors in the pre- and early nCRTs.

Combining pre- and early-treatment MRI data for analysis, unlike in another recent study [29], was another factor that contributed to the robustness of our model. To the best of our knowledge, combining texture analyses at different time points to predict the response of rectal cancer to nCRT has not previously been reported. We found that this combined texture analysis provided a more effective predictor than texture analysis of the pre- or early period only. This combination is reflective of clinical practice and encompasses the diagnosis and treatment process. Most importantly, early-treatment MRI data represent the current status of the tumor after chemoradiotherapy, whereas the data provided by posttreatment MRI scans correspond more closely with pathologic findings in surgical specimens. Hence, including pre- and early-treatment MRI data improves the model's reliability in detecting pCR, potentially enabling clinicians to make a more comprehensive judgment on whether a wait-and-see-treatment approach is warranted.

Our study has some limitations. First place, the small sample size may decrease the generally applicability of our findings. Larger prospective studies are necessary to determine the relevance and robustness of the texture features of rectal cancer. Another limitation is that texture was analyzed only at the level of the largest tumor area and within the fused ROI of upper and lower surfaces, and thus did not represent the overall morphology of the tumor. Unlike solid organ tumors, rectal cancer usually grows along the rectal wall and forms an irregular shape; thus, the whole volume of interest generated from ROIs of consecutive sections may not accurately represent the real shape. Last but not the least, because of time limitations, analysis was performed only of prediction of changes in heterogeneity of tumors; we did not evaluate the predictive value of these texture features for patient survival.

In conclusion, the T2WI image-based texture variables of entropy and energy may be valuable for predicting the efficacy of nCRT in rectal cancer. In addition, early nCRT is the optimal time for predicting response of rectal cancer to nCRT. Texture analysis shows potential as a clinical prognostic tool.

Acknowledgement Fund project of Health Commission of Zhejiang Province, 2019KY302.

Open Access This article is distributed under the terms of the Creative Commons Attribution 4.0 International License (http://creativeco mmons.org/licenses/by/4.0/), which permits unrestricted use, distribution, and reproduction in any medium, provided you give appropriate credit to the original author(s) and the source, provide a link to the Creative Commons license, and indicate if changes were made.

\section{References}

1. Larsen IK, Bray F. Trends in colorectal cancer incidence in Norway 1962-2006: an interpretation of the temporal patterns by anatomic subsite. Int J Cancer, 2010, 126(3): 721-732

2. Sauer R, Liersch T, Merkel S, et al. Preoperative Versus Postoperative Chemoradiotherapy for Locally Advanced Rectal Cancer: Results of the German CAO/ARO/AIO-94 Randomized Phase III Trial After a Median Follow-Up of 11 Years. J Clin Oncol, 2012, 30(16): 1926-1933

3. O'Neill BD, Brown G, Heald RJ, et al. Non-operative treatment after neoadjuvant chemoradiotherapy for rectal cancer. Lancet Oncology. 2007,8(7): 625-633

4. Rodel C, Liersch T, Becker H, et al. Preoperative chemoradiotherapy and postoperative chemotherapy with flurorouracil and oxaliplation versus fluorouracil alone in locally advanced rectal 
cancer: initial results of the German CAO/ARO/AIO-04 randomised phase 3 trial. LANCET ONCOL. 2012, 13(7): 679-687

5. Fernandez-Martos C, Pericay C, Aparicio J, et al. Phase II, randomized study of concomitant chemoradiotherapy followed by surgery and adjuvant capecitabine plus oxaliplatin (CAPOX) compared with induction CAPOX followed by concomitant chemoradiotherapy and surgery in magnetic resonance imaging-defined, locally advanced rectal cancer: Grupo cancer de recto 3 study. J Clin Oncol. 2010, 28(5): 859-865

6. Lim JS, Kim D, Baek SE, et al. Perfusion MRI for the prediction of treatment response after preoperative chemoradiotherapy in locally advanced rectal cancer. Eur Radiol. 2012, 22(8): 1693-1700

7. Monguzzi L, Ippolito D, Bernasconi DP, et al. Locally advanced rectal cancer: value of ADC mapping in prediction of tumor response to radiochemotherapy. Eur J Radiol. 2013, 82(2): 234-240

8. Battersby NJ, Balyasnikova S, Brown G. Guiding post-treatment decisions in rectal cancer: mrTRG is a practical place to start. Oncology(Williston Park). 2014, 28(8): 677-680

9. Garcia-Aguilar J, Pollack J, Lee SH, et al. Accuracy of endorectal ultrasonography in preoperative staging of rectal tumors. Dis Colon Rectum. 2002, 45 (1): 10-15

10. Tylor FG, Quirke P, Heald RJ, et al. Preoperative high-resolution magnetic resonance imaging can identify good prognosis stage I, II, and III rectal cancer best managed by surgery alone: a prospective, multicenter, European study. Ann Surg. 2011, 253(4): 711-719

11. Orphanidou-Vlachou E, Vlachos N, Davies NP, et al. Texture analysis of T1-and T2-weighted MR images and use of probabilistic neural network to discriminate posterior fossa tumours in children[J]. NMR Biomed. 2014, 27(6): 632-639

12. Song I, Kim SH, Lee SJ, et al. Value of diffusion-weighted imaging in the detection of viable tumour after neoadjuvant chemoradiation therapy in patients with locally advanced rectal cancer: comparison with T2 weighted and PET/CT imaging. Br J Radiol. 2012, 85(1013): 577-586

13. Ganeshan B, Goh V, Mandeville HC, et al. Non-small cell lung cancer: histopathologic correlates for texture parameters at CT. Radiology. 2013, 266(1): 326-336

14. Skogen K, Ganeshan B, Good C, et al. Measurements of heterogeneity in gliomas on computed tomography relationship to tumour grade. J Neurooncol. 2013, 111(2): 213-219

15. Dworak O, Keilholz L and Hoffmann A. Pathological features of rectal cancer after preoperative radiochemotherapy. Int J Colorectal Dis. 1997, 12(1): 19-23

16. Vignati A, Mazzetti S, Giannini V, et al. Texture features on T2-weighted magnetic resonance imaging: new potential biomarkers for prostate cancer aggressiveness. Phys Med Biol 2015;60:2685-2701

17. Chee CG, Kim YH, Lee KH,et al.CT texture analysis in patients with locally advanced rectal cancer treated with neoadjuvant chemoradiotherapy: A potential imaging biomarker for treatment response and prognosis. PLoS One. 2017,12(8):e0182883

18. Liu L, Liu Y, Xu L,et al.Application of texture analysis based on apparent diffusion coefficient maps in discriminating different stages of rectal cancer.J Magn Reson Imaging. 2017, 45(6): 1798-1808

19. Hunter C, Siddiqui M, Georgiou Delisle T, et al. CT and 3-T MRI accurately identify $\mathrm{T} 3 \mathrm{c}$ disease in colon cancer, which strongly predicts disease-free survival. Clin Radiol. 2017, 72(4):307-315

20. Davnall F, Yip CS, Ljungqvist G, et al. Assessment of tumor heterogeneity:an emerging imaging tool for clinical practice? Insights Imaging. 2012, 3(6): 573-589

21. De Cecco CN, Ciolina M, Caruso D, et al.Performance of diffusion-weighted imaging, perfusion imaging, and texture analysis in predicting tumoral response to neoadjuvant chemoradiotherapy in rectal cancer patients studied with 3T MR: initial experience. Abdom Radiol (NY). 2016, 41(9): 1728-35

22. Alobaidli S, McQuaid S, South C, et al. The role of texture analysis in imaging as an outcome predictor and potential tool in radiotherapy treatment planning. Br J Radiol. 2014, 87(1042): 20140369

23. De Cecco CN, Ganeshan B, Ciolina M, et al. Texture analysis as imaging biomarker of tumoral response to neoadjuvant chemoradiotherapy in rectal cancer patients studied with 3-T magnetic resonance. Investigative radiology. 2015, 50(4): 239-245

24. Hegde JV, Mulkern RV, Panych LP et al. Multiparametric MRI of prostate cancer: an update on state-of-the-art techniques and their performance in detecting and localizing prostate cancer. J Magn Reson Imaging. 2013, 37: 1035-1054

25. Ahmed A, Gibbs P, Pickles M, Turnbull L. Texture analysis in assessment and prediction of chemotherapy response in breast cancer. J Magn Reson Imaging. 2013, 38(1): 89-101

26. Miles KA, Ganeshan B, Hayball MP. CT texture analysis using the filtration histogram method: what do the measurements mean? Cancer Imaging. 2013, 13(3): 400-406

27. Duvauferrier R, Bezy J, Bertaud V, et al. Texture analysis software: integration with a radiological workstation. Stud Health Technol Inform. 2012, 180: 1030-1034

28. Liu L, Liu Y, Xu L, et al. Application of Texture Analysis Based on Apparent Diffusion Coefficient Maps in Discriminating Different Stages of Rectal Cancer. J Magn Reson Imaging. 2017, 45(6): 1798-1808

29. Wang G, He L, Yuan C,et al. Pretreatment MR imaging radiomics signatures for response prediction to induction chemotherapy in patients with nasopharyngeal carcinoma. Eur J Radiol. 2018, 98 : 100-106

Publisher's Note Springer Nature remains neutral with regard to jurisdictional claims in published maps and institutional affiliations. 